\title{
QUEEN'S
UNIVERSITY
BELFAST
}

\section{Two-Stage Limited Feedback Hybrid Precoding in Multiuser MIMO Systems}

Almradi, A., Matthaiou, M., \& Fusco, V. F. (2020). Two-Stage Limited Feedback Hybrid Precoding in Multiuser MIMO Systems. In 2020 IEEE International Conference on Communications: Proceedings (ICC: IEEE International Conference on Communications (ICC): Proceedings). Institute of Electrical and Electronics Engineers Inc.. https://doi.org/10.1109/ICC40277.2020.9149364

Published in:

2020 IEEE International Conference on Communications: Proceedings

Document Version:

Peer reviewed version

Queen's University Belfast - Research Portal:

Link to publication record in Queen's University Belfast Research Portal

Publisher rights

Copyright 2020 IEEE. This work is made available online in accordance with the publisher's policies. Please refer to any applicable terms of use of the publisher.

\section{General rights}

Copyright for the publications made accessible via the Queen's University Belfast Research Portal is retained by the author(s) and / or other copyright owners and it is a condition of accessing these publications that users recognise and abide by the legal requirements associated with these rights.

Take down policy

The Research Portal is Queen's institutional repository that provides access to Queen's research output. Every effort has been made to ensure that content in the Research Portal does not infringe any person's rights, or applicable UK laws. If you discover content in the Research Portal that you believe breaches copyright or violates any law, please contact openaccess@qub.ac.uk. 


\title{
Two-Stage Limited Feedback Hybrid Precoding in Multiuser MIMO Systems
}

\author{
Ahmed Almradi, Michail Matthaiou, and Vincent F. Fusco \\ Institute of Electronics, Communications and Information Technology (ECIT), Queen's University Belfast, U.K. \\ Emails: \{a.almradi, m.matthaiou\}@qub.ac.uk, and v.fusco@ecit.qub.ac.uk.
}

\begin{abstract}
Accurate channel state information (CSI) is essential at the base station (BS) in order to serve multiple users simultaneously on the same time-frequency resources. Explicit low-rate CSI feedback from each user terminal to the BS is indispensable in frequency-division duplexing (FDD) multipleinput multiple-output (MIMO) hybrid precoding systems. In the presence of correlated Rician fading channels with arbitrary rank, and in order to reduce the feedback overhead, a unified adaptive statistical codebook is designed by leveraging the long-term first and second order channel statistics. Moreover, as the feedback overhead scales linearly with the number of BS antennas, which introduces a bottleneck in FDD massive MIMO systems, an adaptive two-stage reduced dimensionality codebook design is developed. The main idea stems from the fact that the high dimensional analog beamforming stage could get away with coarse CSI, e.g., statistical CSI, while the low dimensional baseband precoding stage acquires finite-rate quantized CSI of the reduced dimensionality channel to eliminate multiuser interference. These steps reduce significantly the CSI training and feedback overhead to scale linearly with the number of radio-frequency chains instead. For comparison purposes, the two extremes of full and statistical CSI at the $\mathrm{BS}$ are also included. The performance gap between these two extremes justifies the importance of the proposed two-stage reduced dimensionality adaptive statistical codebook.
\end{abstract}

\section{INTRODUCTION}

$\mathbf{M}$ ASSIVE multiple-input multiple-output (MIMO) systems have emerged as one of the essential components of the fifth-generation $(5 \mathrm{G})$ and beyond wireless communications [1]-[4]. However, a dedicated radiofrequency $(\mathrm{RF})$ chain is needed for each antenna element in the conventional fully digital structure, which results in enormous cost and power consumption. To alleviate these issues, hybrid analog and digital precoding transceivers have been lately investigated, where a much smaller number of $\mathrm{RF}$ chains are connected to a massive number of antennas via a set of low power and cost analog phase shifters. These types of architectures divide the precoding and beamforming stages between the digital baseband and analog RF domains, respectively [1], [2], [5].

To completely harvest the advantages of multiuser MIMO systems, perfect channel state information at the transmitter (CSIT) is indispensable in order to accurately leverage the idea of space division multiple access (SDMA) [2]. However, channel state information (CSI) acquisition and feedback overhead is the bottleneck of massive MIMO

This work was supported by the U.K. Engineering and Physical Sciences Research Council (EPSRC) under Grant EP/P000673/1. systems in frequency-division duplexing (FDD) systems. Unlike time-division duplexing (TDD) systems, where the reciprocity of the channel is being leveraged, explicit finiterate quantized CSI feedback from each user terminal to the BS is essential in FDD systems. ${ }^{1}$ Hence, the downlink training and uplink feedback overhead resources have to scale linearly with the number of BS antennas in order to control the estimation and quantization errors [6], [7]. Therefore, channel dimensionality reduction techniques are crucial for the feasibility of FDD in massive MIMO systems.

In the context of fully digital structures, a two-stage beamformer called joint spatial division and multiplexing (JSDM) was proposed in [7], where users are divided into groups based on the similarities of their second-order channel statistics, then a two-stage precoding is designed. Motivated by the idea of JSDM, the authors in [8] introduced an antenna grouping technique that substantially reduces the feedback overhead in FDD massive MIMO systems. In the context of hybrid precoding, the authors in [4] designed the analog beamformer based only on the channel covariance matrices of the users, utilizing the averaged signal-to-leakage-plus-noise ratio (SLNR) metric, while the digital precoder is determined by using the regularized zero-forcing (ZF) technique, exploiting full CSIT of the reduced dimensionality effective channel. However, acquiring perfect CSI for the effective channel at the BS is infeasible as an effective channel quantization codebook with infinite resolution is required.

In finite-rate limited feedback channels, the channel is quantized using a pre-defined codebook that is known at both the BS and user terminals. Then, each user terminal feeds back a finite number of $B$ bits indicating the codeword index that is closest to the actual channel. The codebook design problem was first considered for channels with hypersphere subspace, i.e., for independent and identically distributed (i.i.d.) Rayleigh fading channels (see e.g., [6]), via isotropic random vector quantization (RVQ) codebook. In [9], a skewed codebook design was proposed for correlated Rayleigh fading channels, using a modified RVQ codebook with hyperellipse channel subspace that is skewed according to the second order channel statistics. In [2], a two-stage feedback scheme was proposed for hybrid precod-

\footnotetext{
${ }^{1}$ In FDD systems, the uplink and downlink channels have to be estimated separately as they operate in different frequency bands. Therefore, in order to have accurate CSIT in FDD systems, the channel has to be estimated at each user terminal with the aid of training symbols and then fed back to the BS via a finite low-rate feedback channel.
} 
ing in multiuser MIMO, where the analog beamformer and baseband precoder are designed based on the quantization and feedback of a beamsteering and an isotropic RVQ codebooks, respectively. The authors in [3] proposed a one-stage feedback scheme where all feedback overhead is utilized for the quantization and feedback of the analog beamformer, while leveraging only the second order channel statistics for the design of the digital precoder. Furthermore, in contrast to [2], under a fixed total feedback overhead constraint, the authors in [3] also developed a two-stage feedback scheme through which the analog beamformer and baseband precoder are designed based on the quantization and feedback of an RF beamsteering and a skewed RVQ codebooks with $B_{R F}$ and $B_{B B}$ bits, respectively, where $B=B_{R F}+B_{B B}$ is the total number of feedback bits. Finally, motivated by the slowly varying nature of the path angle-of-departures (AoD), the authors in [10] proposed an AoD based adaptive codebook for FDD massive MIMO systems.

Most previously investigated codebooks are designed based on the assumption of correlated Rayleigh fading channels and cannot be easily generalized to scenarios where we have a dominant line-of-sight (LOS) path. This generalized model is suitable for dense small cell networks, vehicular networks, indoor applications, and millimeter wave (mmWave) propoagation. In addition, the feedback overhead of previously investigated codebooks scales linearly with the number of BS antennas, introducing a bottleneck in FDD massive MIMO systems. Therefore, in correlated Rician fading channels with arbitrary rank, which covers both rich and sparse scattering channels, a more relevant codebook design is pivotal. To the best of our knowledge, no work exists on the design of adaptive channel statistics codebooks for Rician fading channels. To this end, we first propose a unified adaptive statistical codebook design for correlated Rician fading channels by leveraging the channel statistics information. Second, due to the overwhelming CSI training and feedback overhead in FDD massive MIMO, a reduced dimensionality twostage adaptive statistical codebook design is developed, where the high dimensional analog beamformer leverages only the long-term first and second order channel statistics, while the low dimensional digital precoder acquires finiterate quantized CSI for the reduced dimensionality effective channel, thus substantially reducing the training and feedback overhead to scale linearly with the number of active users. The proposed two-stage CSIT scheme uses the SLNR metric to jointly design the analog beamformer and baseband precoder based on statistical and quantized CSIT, respectively.

\section{System And Channel Model}

Consider a single-cell multiuser MIMO downlink system, where a BS equipped with $N_{B S}$ antennas and $N_{R F}$ RF chains serves $K$ single antenna users, where $K \leq N_{R F} \leq$ $N_{B S}$. A hybrid analog RF beamformer $\boldsymbol{F} \in \mathbb{C}^{N_{B S} \times N_{R F}}$ and a digital baseband precoder $\boldsymbol{W} \in \mathbb{C}^{N_{R F} \times K}$ are deployed at the BS as illustrated in Fig. 1. For simplicity,

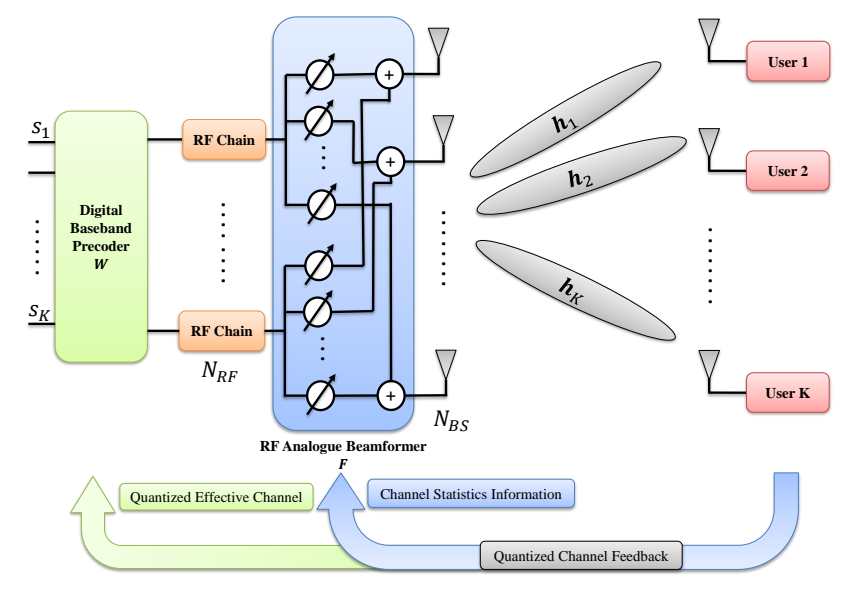

Figure 1: The two-stage hybrid multiuser MIMO system with limited feedback.

it is assumed that $N_{R F}=K$. As a network of phase shifters is used to represent the analog RF beamforming stage, its entries are restricted to have a constant modulus, i.e., $\left|[\boldsymbol{F}]_{m, n}\right|=\frac{1}{\sqrt{N_{B S}}}$, where $|\cdot|$ denotes the absolute value operator.

To explicitly take into account the LOS component, a correlated Rician fading channel with arbitrary rank is considered, hence, the channel between the $k^{\text {th }}$ user and the BS is expressed as ${ }^{2}$

$$
\boldsymbol{h}_{k}=\alpha_{k} \boldsymbol{h}_{\mathrm{LOS}, k}+\beta_{k} \boldsymbol{U}_{k} \boldsymbol{\Lambda}_{k}^{\frac{1}{2}} \boldsymbol{h}_{\mathrm{NLOS}, k},
$$

where $\alpha_{k}=\sqrt{\frac{K_{k}}{K_{k}+1}}, \beta_{k}=\sqrt{\frac{1}{K_{k}+1}}, K_{k}$ is the ratio between the specular LOS and scattered non-LOS channel components, known as the Rician K-factor for the $k^{\text {th }}$ user, $\boldsymbol{\Sigma}_{k}=\boldsymbol{U}_{k} \boldsymbol{\Lambda}_{k} \boldsymbol{U}_{k}^{\dagger}$ is the eigenvalue decomposition for the $k^{\text {th }}$ user's channel covariance matrix, $\boldsymbol{\Lambda}_{k}$ is an $r_{k} \times r_{k}$ diagonal matrix whose entries are the $r_{k}$ nonzero dominant eigenvalues of $\boldsymbol{\Sigma}_{k}$, with arbitrary $\operatorname{rank}^{3}$ of $r_{k}=\operatorname{rank}\left(\boldsymbol{\Sigma}_{k}\right)$, $\boldsymbol{U}_{k} \in \mathbb{C}^{N_{B S} \times r_{k}}$ is a semi-unitary matrix consists of the eigenvectors of $\boldsymbol{\Sigma}_{k}$ corresponding to the nonzero eigenvalues, $\boldsymbol{h}_{\mathrm{NLOS}, k} \in \mathbb{C}^{r_{k} \times 1}$ denotes the random non-LOS channel for the $k^{\text {th }}$ user, whose entries are i.i.d. random variables and distributed according to $\mathcal{C N}(0,1)$, while $\boldsymbol{h}_{\mathrm{LOS}, k} \in \mathbb{C}^{N_{B S} \times 1}$ represents the deterministic (LOS) component, satisfying $\left\|\boldsymbol{h}_{\mathrm{LOS}, k}\right\|^{2}=N_{B S}$, where $\|\cdot\|$ denotes the Euclidean norm operator, defined as

$$
\boldsymbol{h}_{\mathrm{LOS}, k}=\left[1, e^{-j \frac{2 \pi d}{\lambda} \sin \left(\varphi_{k}\right)}, \ldots, e^{-j \frac{2 \pi d}{\lambda}\left(N_{B S}-1\right) \sin \left(\varphi_{k}\right)}\right]^{T}
$$

where $\lambda$ is the wavelength, $d$ is the inter-antenna spacing, and $\varphi_{k}$ is the $k^{\text {th }}$ user AoD, which is uniformly distributed according to $\varphi_{k} \sim \mathcal{U}\left(-\frac{\pi}{2}, \frac{\pi}{2}\right)$.

\footnotetext{
${ }^{2}$ The correlated Rayleigh fading channel model introduced in [7, Eq. (2)] is generalized here for the case where there is a strong LOS component.

${ }^{3}$ The rank of the channel covariance matrix depends on the angular spread, inter-antenna spacing, and wavelength [7]. For instance, from (25), if we set $N_{B S}=64, d=0.5 \lambda, \phi_{k}=\frac{\pi}{6}$, and $\Delta_{k}=15^{\circ}$, these settings result in a covariance matrix of rank $r_{k}=38$.
} 
The composite transmitted signal and the received signal at the $k^{\text {th }}$ user are respectively given as

$$
\begin{gathered}
\boldsymbol{x}=\boldsymbol{F} \boldsymbol{W} \boldsymbol{P} \boldsymbol{s}=\sum_{j=1}^{K} \sqrt{P_{j}} \boldsymbol{F} \boldsymbol{w}_{j} s_{j}, \\
y_{k}=\underbrace{\sqrt{P_{k}} \boldsymbol{h}_{k}^{\dagger} \boldsymbol{F} \boldsymbol{w}_{k} s_{k}}_{\text {desired signal }}+\underbrace{\boldsymbol{h}_{k}^{\dagger} \sum_{j \neq k} \sqrt{P_{j}} \boldsymbol{F} \boldsymbol{w}_{j} s_{j}}_{\text {multiuser interference }}+\underbrace{n_{k}}_{\text {AWGN noise }},
\end{gathered}
$$

where $s \in \mathbb{C}^{K \times 1}=\left[s_{1}, s_{2}, \ldots, s_{K}\right]^{T}$ are the payload data symbols intended for the $K$ scheduled users, with entries that are i.i.d. Gaussian random variables with zero mean and unit variance, $n_{k}$ is the additive white Gaussian noise (AWGN) at the $k^{\text {th }}$ user, distributed as $n_{k} \sim \mathcal{C N}\left(0, \sigma^{2}\right)$, and $\boldsymbol{P}=\operatorname{diag}\left(P_{1}, P_{2}, \ldots, P_{K}\right)$ is a diagonal power allocation matrix that satisfies the power constraint, i.e., the total transmit power $\sum_{j=1}^{K} P_{j}=P$. For ease of exposition, the power is equally allocated amongst users, i.e., $\boldsymbol{P}=\sqrt{\frac{P}{K}} \boldsymbol{I}_{K}$, hence, in this work, we normalize the analog and digital precoders such that $\left\|\boldsymbol{F} \boldsymbol{w}_{j}\right\|^{2}=1$, where $\|\cdot\|$ denotes the Euclidean norm, and $\boldsymbol{I}_{K}$ is the identity matrix of size $K \times K$.

Therefore, from (4), the signal-to-interference-plus-noise ratio (SINR) for the $k^{\text {th }}$ user is written as

$$
\operatorname{SINR}_{k}=\frac{\rho\left|\boldsymbol{h}_{k}^{\dagger} \boldsymbol{F} \boldsymbol{w}_{k}\right|^{2}}{1+\rho \sum_{j \neq k}\left|\boldsymbol{h}_{k}^{\dagger} \boldsymbol{F} \boldsymbol{w}_{j}\right|^{2}}
$$

where $\rho=\frac{P}{K \sigma^{2}}$ is the signal-to-noise ratio (SNR).

The achievable sum spectral efficiency of the system is expressed as

$$
\mathcal{R}_{\text {sum }}=\sum_{k=1}^{K} \mathcal{R}_{k}=\sum_{k=1}^{K} \mathbb{E}\left[\log _{2}\left(1+\operatorname{SINR}_{k}\right)\right]
$$

where $\mathbb{E}[\cdot]$ denotes the expectation operator.

Our aim is to find closed-form solutions for $\boldsymbol{F}$ and $\boldsymbol{W}$ such that the spectral efficiency in (6) is maximized. However, due to the coupling between the precoders of all users $\left\{\boldsymbol{w}_{k}, k=1, \ldots, K\right\}$ in (5), deriving closed-form solutions are challenging because of the non-convex nature of the optimization problem. Consequently, maximum ratio transmission (MRT) and $\mathrm{ZF}$ schemes are two well known approaches exploited to attain sub-optimal closedform beamformers and precoders [2], [11]. Contrary, in this paper, to avoid tackling the coupled optimization problem in (6), we leverage the SLNR metric, which results in decoupling the precoders, thus leading to a near-optimal closed-form solution [3], [4], [12], [13].

The SLNR for the $k^{\text {th }}$ user is defined as [13]

$$
\mathrm{SLNR}_{k}=\frac{\rho\left|\boldsymbol{h}_{k}^{\dagger} \boldsymbol{F} \boldsymbol{w}_{k}\right|^{2}}{1+\rho \sum_{j \neq k}\left|\boldsymbol{h}_{j}^{\dagger} \boldsymbol{F} \boldsymbol{w}_{k}\right|^{2}} .
$$

Let $\boldsymbol{V}$ be the unconstrained analog beamformer, where the amplitude and phase of its entries can be controlled, then the constrained analog beamformer $\boldsymbol{F}$ can be derived as [4]

$$
\left[\boldsymbol{F}^{\star}\right]_{m, n}=\frac{1}{\sqrt{N_{B S}}} \exp \left(\measuredangle[\boldsymbol{V}]_{m, n}\right) .
$$

Therefore, in this paper, the optimal unconstrained analog beamformer $\boldsymbol{V}$ is derived first, through which the constrained analog beamformer $\boldsymbol{F}$ is then determined by (8).

\section{CSIT EXTREMES}

\section{A. Full CSIT}

Having fewer number of RF chains than BS antennas makes it infeasible to acquire full CSIT in both TDD and FDD systems. Therefore, the full CSIT is considered here simply for benchmarking against the proposed adaptive statistical codebooks. Thus, in this section, the analog beamformer $\boldsymbol{V}$ and baseband precoder $\boldsymbol{W}$ are both designed based on perfect instantaneous CSIT at the BS.

The $k^{\text {th }}$ user's optimal baseband precoder, which is formulated as $\boldsymbol{w}_{k}^{\star}=\underset{\left\|\boldsymbol{V} \boldsymbol{w}_{k}\right\|^{2}=1}{\arg \max } \mathrm{SLNR}_{k}$, is the generalized eigenvector, given by [13, Eq. (11)]

$$
\boldsymbol{w}_{k}^{\star}=\frac{\left(\boldsymbol{V}^{\dagger} \boldsymbol{V}+\rho \sum_{j \neq k} \boldsymbol{V}^{\dagger} \boldsymbol{h}_{j} \boldsymbol{h}_{j}^{\dagger} \boldsymbol{V}\right)^{-1} \boldsymbol{V}^{\dagger} \boldsymbol{h}_{k}}{\left\|\boldsymbol{V}\left(\boldsymbol{V}^{\dagger} \boldsymbol{V}+\rho \sum_{j \neq k} \boldsymbol{V}^{\dagger} \boldsymbol{h}_{j} \boldsymbol{h}_{j}^{\dagger} \boldsymbol{V}\right)^{-1} \boldsymbol{V}^{\dagger} \boldsymbol{h}_{k}\right\|} .
$$

The optimal $k^{\text {th }}$ column of the analog beamformer $\boldsymbol{v}_{k}^{\star}$, which is formulated as $\boldsymbol{v}_{k}^{\star}=\arg \max \mathrm{SLNR}_{k}$, is derived as

$$
\boldsymbol{v}_{k}^{\star}=\left(\boldsymbol{I}_{N_{B S}}+\rho \sum_{j \neq k} \boldsymbol{h}_{j} \boldsymbol{h}_{j}^{\dagger}\right)^{-1} \boldsymbol{h}_{k} .
$$

Proof: The proof is given in Appendix A.

\section{B. Statistical CSIT}

Statistical CSIT is more desirable than full CSIT in FDD massive MIMO as it varies over a longer timescale when compared to the instantaneous channel variations, hence, substantially reducing the required training sequences and feedback overhead. However, a residual amount of multiuser interference remains due to the impact of unknown instantaneous multipath components, resulting in a performance saturation particularly at high SNR. Therefore, in this section, the analog beamformer $\boldsymbol{V}$ and baseband precoder $\boldsymbol{W}$ are designed based only on the long-term first and second order channel statistics information via the maximization of a lower-bound on the average SLNR.

A lower-bound on the average SLNR for the $k^{\text {th }}$ user is written by 4

$$
\mathbb{E}\left[\mathrm{SLNR}_{k}\right] \geq \frac{\rho \boldsymbol{w}_{k}^{\dagger} \boldsymbol{V}^{\dagger} \boldsymbol{R}_{k} \boldsymbol{V} \boldsymbol{w}_{k}}{\boldsymbol{w}_{k}^{\dagger}\left(\boldsymbol{V}^{\dagger} \boldsymbol{V}+\rho \sum_{j \neq k} \boldsymbol{V}^{\dagger} \boldsymbol{R}_{j} \boldsymbol{V}\right) \boldsymbol{w}_{k}},
$$

${ }^{4}$ The derivation of a lower-bound on $\mathbb{E}\left[\mathrm{SLNR}_{k}\right]$ follows similar steps to [3, Eq. (10)]. 
where $\boldsymbol{R}_{k}=\alpha_{k}^{2} \boldsymbol{h}_{\mathrm{LOS}, k} \boldsymbol{h}_{\mathrm{LOS}, k}^{\dagger}+\beta_{k}^{2} \boldsymbol{\Sigma}_{k}$

Therefore, the $k^{\text {th }}$ user's optimal baseband precoder, which is formulated as $\boldsymbol{w}_{k}^{\star}=\arg \max \mathbb{E}\left[\mathrm{SLNR}_{k}\right]$, is given

by [3, Eq. (11)]

$$
\boldsymbol{w}_{k}^{\star}=\frac{\boldsymbol{u}_{\max }\left[\left(\boldsymbol{V}^{\dagger} \boldsymbol{V}+\rho \sum_{j \neq k} \boldsymbol{V}^{\dagger} \boldsymbol{R}_{j} \boldsymbol{V}\right)^{-1} \boldsymbol{V}^{\dagger} \boldsymbol{R}_{k} \boldsymbol{V}\right]}{\left\|\boldsymbol{V} \boldsymbol{u}_{\max }\left[\left(\boldsymbol{V}^{\dagger} \boldsymbol{V}+\rho \sum_{j \neq k} \boldsymbol{V}^{\dagger} \boldsymbol{R}_{j} \boldsymbol{V}\right)^{-1} \boldsymbol{V}^{\dagger} \boldsymbol{R}_{k} \boldsymbol{V}\right]\right\|_{(12)}},
$$

where $\boldsymbol{u}_{\max }[\boldsymbol{X}]$ is the dominant eigenvector of the matrix $\boldsymbol{X}$.

Substituting (12) into (11), reduces to

$\mathbb{E}\left[\mathrm{SLNR}_{k}\right] \geq \rho \lambda_{\max }\left[\left(\boldsymbol{V}^{\dagger} \boldsymbol{V}+\rho \sum_{j \neq k} \boldsymbol{V}^{\dagger} \boldsymbol{R}_{j} \boldsymbol{V}\right)^{-1} \boldsymbol{V}^{\dagger} \boldsymbol{R}_{k} \boldsymbol{V}\right]$,

where $\lambda_{\max }[\boldsymbol{X}]$ is the strongest eigenvalue of the matrix $\boldsymbol{X}$.

Hence, the optimal $k^{\text {th }}$ column of the analog beamformer $\boldsymbol{v}_{k}^{\star}$, which is formulated as $\boldsymbol{v}_{k}^{\star}=\arg \max \mathbb{E}\left[\mathrm{SLNR}_{k}\right]$, is written as

$$
\boldsymbol{v}_{k}^{\star}=\boldsymbol{u}_{\max }\left[\left(\boldsymbol{I}_{N_{B S}}+\rho \sum_{j \neq k} \boldsymbol{R}_{j}\right)^{-1} \boldsymbol{R}_{k}\right] .
$$

\section{QUANTIZED CSIT}

In FDD systems, each downlink channel vector $\boldsymbol{h}_{k}$ is estimated at the user terminal through a dedicated downlink training sequences that scales linearly with the number of BS antennas. It is assumed here that each user knows perfectly its channel [3], [8], [10]. Therefore, with the help of a pre-defined codebook that is shared between the BS and user terminals, each user needs to quantize its channel to the nearest codeword in the codebook. Due to the limited system resources, this codeword is fed back to the BS via a low-rate limited feedback channel. The user specific codebook $\mathcal{C}_{k}=\left\{\boldsymbol{c}_{k, 1}, \boldsymbol{c}_{k, 2}, \ldots, \boldsymbol{c}_{k, 2^{B}}\right\}$ comprises $2^{B} \quad N_{B S}$-dimensional unit norm vectors, where $B$ is the available finite number of feedback bits. The channel quality information (CQI) $\left\|\boldsymbol{h}_{k}\right\|$ is assumed to be perfectly known at the $\mathrm{BS},{ }^{5}$ while the channel direction information (CDI) $\widetilde{\boldsymbol{h}}_{k}=\frac{\boldsymbol{h}_{k}}{\left\|\boldsymbol{h}_{k}\right\|}$ is quantized to the nearest neighbor codeword $\boldsymbol{c}_{k, \pi(k)}$ in the codebook $\mathcal{C}_{k}$, where the quantization (codeword) index for the $k^{\text {th }}$ user $\pi(k)$ is given as

$$
\pi(k)=\underset{\boldsymbol{c}_{k, i} \in \mathcal{C}_{k}}{\arg \max }\left|\widetilde{\boldsymbol{h}}_{k}^{\dagger} \boldsymbol{c}_{k, i}\right|^{2} .
$$

The quantization index for the $k^{\text {th }}$ user $\pi(k)$ is then fed back to the BS via a finite-rate of $B$ feedback bits per user. The BS utilizes this index to obtain the quantized CSIT that is given by $\widehat{\boldsymbol{h}}_{k}=\left\|\boldsymbol{h}_{k}\right\| \boldsymbol{c}_{k, \pi(k)}$. Thus, the analog beamformer $\boldsymbol{V}$ and baseband precoder $\boldsymbol{W}$ here are designed

\footnotetext{
${ }^{5}$ Note that the quantization and feedback of the scalar value $\left\|\boldsymbol{h}_{k}\right\|$ can be easily and accurately performed with very few number of bits and low rate feedback when compared to the requirements of massive dimensional vectors (massive MIMO channels) [3], [8], [10].
}

based on the quantized CSIT $\widehat{\boldsymbol{H}}=\left[\widehat{\boldsymbol{h}}_{1}, \widehat{\boldsymbol{h}}_{1}, \ldots, \widehat{\boldsymbol{h}}_{K}\right]$ at the BS.

Therefore, from (4), and based on the quantized fed back CSIT, the SLNR for the $k^{\text {th }}$ user is defined as

$$
\operatorname{SLNR}_{k}=\frac{\rho \boldsymbol{w}_{k}^{\dagger} \boldsymbol{V}^{\dagger} \widehat{\boldsymbol{h}}_{k} \widehat{\boldsymbol{h}}_{k}^{\dagger} \boldsymbol{V} \boldsymbol{w}_{k}}{\boldsymbol{w}_{k}^{\dagger}\left(\boldsymbol{V}^{\dagger} \boldsymbol{V}+\rho \sum_{j \neq k} \boldsymbol{V}^{\dagger} \widehat{\boldsymbol{h}}_{j} \widehat{\boldsymbol{h}}_{j}^{\dagger} \boldsymbol{V}\right) \boldsymbol{w}_{k}} .
$$

Following similar derivations to [13, Eq. (11)], the $k^{\text {th }}$ user's optimal baseband precoder is given as

$$
\boldsymbol{w}_{k}^{\star}=\frac{\left(\boldsymbol{V}^{\dagger} \boldsymbol{V}+\rho \sum_{j \neq k} \boldsymbol{V}^{\dagger} \widehat{\boldsymbol{h}}_{j} \widehat{\boldsymbol{h}}_{j}^{\dagger} \boldsymbol{V}\right)^{-1} \boldsymbol{V}^{\dagger} \widehat{\boldsymbol{h}}_{k}}{\left\|\boldsymbol{V}\left(\boldsymbol{V}^{\dagger} \boldsymbol{V}+\rho \sum_{j \neq k} \boldsymbol{V}^{\dagger} \widehat{\boldsymbol{h}}_{j} \widehat{\boldsymbol{h}}_{j}^{\dagger} \boldsymbol{V}\right)^{-1} \boldsymbol{V}^{\dagger} \widehat{\boldsymbol{h}}_{k}\right\|_{(1)}} .
$$

The optimal $k^{\text {th }}$ column of the analog beamformer $\boldsymbol{v}_{k}^{\star}$ is written as

$$
\boldsymbol{v}_{k}^{\star}=\left(\boldsymbol{I}_{N_{B S}}+\rho \sum_{j \neq k} \widehat{\boldsymbol{h}}_{j} \widehat{\boldsymbol{h}}_{j}^{\dagger}\right)^{-1} \widehat{\boldsymbol{h}}_{k}
$$

In the finite-rate feedback settings, the achievable sum spectral efficiency in (6) relies on the quantized CSIT through the analog beamformer $\boldsymbol{V}$ and baseband precoder $\boldsymbol{W}$ in (18) and (17), respectively.

\section{Statistical Codebook Design}

The codebook design depends heavily on the channel statistics and distribution. For instance, from the current literature, in pure LOS channels, if the $k^{\text {th }}$ user AoD is uniformly distributed according to $\varphi_{k} \sim \mathcal{U}(0, \pi)$, a beamsteering discrete Fourier transform (DFT) codebook was proposed $\mathcal{F}=\left\{f_{i}=\mathbf{a}\left(\frac{\pi}{2^{B}} i\right), i=1, \ldots, 2^{B}\right\}$, where $\mathbf{a}\left(\varphi_{k}\right)$ is given similarly to (2) [2], [3]. Meanwhile, a RVQ codebook was proposed for i.i.d. uncorrelated Raleigh fading channels in [6], where a common isotropically distributed codebook $\mathcal{V}_{\text {i.i.d. }}=\left\{\boldsymbol{v}_{1}, \boldsymbol{v}_{2}, \ldots, \boldsymbol{v}_{2^{B}}\right\}$, with $\boldsymbol{v}_{i} \sim \mathcal{C N}\left(0, \boldsymbol{I}_{N_{B S}}\right)$ normalized such that $\left\|\boldsymbol{v}_{i}\right\|=1$. This codebook is common for all users, assuming that the channel subspace is a hypersphere in $\mathbb{C}^{N_{B S}}$. On the other hand, an adaptive per-user codebook design was proposed for correlated Rayleigh fading channels in [9], where the authors introduced a modified skewed RVQ codebook $\mathcal{W}_{k}=\left\{\boldsymbol{w}_{k, i}=\frac{\boldsymbol{\Sigma}_{k}^{\frac{1}{2}} \boldsymbol{v}_{i}}{\left\|\boldsymbol{\Sigma}_{k}^{\frac{1}{2}} \boldsymbol{v}_{i}\right\|}, i=1, \ldots, 2^{B}\right\}$ with a hyperellipses channel subspace skewed by the eigenvalues of $\boldsymbol{\Sigma}_{k}$, where $\boldsymbol{\Sigma}_{k}$ is the $k^{\text {th }}$ user covariance matrix. Therefore, the channel codebook design problem is simply a channel subspace vector quantization problem, where the choice of the channel quantization codebook has a significant impact on the quality of the quantized channel.

The codebook design metric is defined as a distortion metric, given by

$$
\mathcal{D}\left(\mathcal{C}_{k}\right)=\mathbb{E}\left[\left\|\boldsymbol{h}_{k}\right\|^{2}-\left\|\boldsymbol{h}_{k}\right\|^{2} \max _{\boldsymbol{c}_{k, i} \in \mathcal{C}_{k}}\left|\widetilde{\boldsymbol{h}}_{k}^{\dagger} \boldsymbol{c}_{k, i}\right|^{2}\right],
$$

where $\left\|\boldsymbol{c}_{k, i}\right\|=1$. 
The design objective of a quantized channel is to intelligently construct a channel statistics codebook so as the distortion metric in (19) is minimized. This is done by properly quantizing only the channel subspace, i.e., finely quantizing the channel subspace according to the channel statistics and distribution.

In multiuser MIMO systems, to guarantee a constant spectral efficiency rate gap between full and quantized CSIT, the number of feedback bits needs to scale linearly with the rank of the channel and SNR (in decibels), according to $B=O\left(\frac{r_{k}-1}{3} \rho_{\mathrm{dB}}\right)$. For instance, $B=50$ feedback bits are needed in rich scattering channels to guarantee a constant gap when $N_{B S}=16$, and $\rho_{\mathrm{dB}}=10 \mathrm{~dB}$ [6], [8], [10]. Therefore, in order to reduce the amount of feedback required to attain a fixed rate gap in FDD massive MIMO, two critical issues need to be addressed: 1) What is the optimal codebook structure that accurately quantizes the channel subspace, and 2) How to design ingenious dimensionality reduction techniques that substantially reduce the CSI acquisition and feedback overhead.

\section{A. Proposed Adaptive Statistical Codebook}

In this section, we introduce a skewed and shifted RVQ codebook for correlated Rician fading channels with arbitrary rank. According to the maximal-ratio combining (MRC) principle, it is well know that with infinite resolution codebook, the optimal codeword is given as $\boldsymbol{c}_{k}^{\mathrm{opt}}=\underset{\boldsymbol{c}_{k}}{\arg \max }\left|\widetilde{\boldsymbol{h}}_{k}^{\dagger} \boldsymbol{c}_{k}\right|^{2}=\widetilde{\boldsymbol{h}}_{k}$, which from (19) results in a zero distortion. Therefore, in order to maximize the average projection of the channel direction onto the codwords $\left|\widetilde{\boldsymbol{h}}_{k}^{\dagger} \boldsymbol{c}_{k, i}\right|$ so as to minimize the distortion in (19), the channel and codewords need to lie in the same subspace and have the same statistical distribution, i.e., if the channel fading is distributed according to correlated Rician fading $\boldsymbol{h}_{k} \sim \mathcal{C N}\left(\boldsymbol{\mu}_{k}, \boldsymbol{\Sigma}_{k}\right)$, then the optimal codebook design structure needs to quantize only this channel subspace accurately, as any codewords outside the channel subspace would not be used due to its high projection loss. Therefore, to optimally utilize all the limited finite number of $B$ bits, the optimal codebook has to finely quantize the channel subspace by mimicking the channel statistical distribution, i.e., $\boldsymbol{c}_{k, i} \sim \mathcal{C N}\left(\boldsymbol{\mu}_{k}, \boldsymbol{\Sigma}_{k}\right)$. With this setting, the codewords will perfectly cover the channel subspace with the least possible codebook size and projection loss (distortion). Consequently, the proposed adaptive statistical codebook is derived by leveraging the channel mean and covariance statistics, as follows

$$
\mathcal{C}_{k}=\left\{\boldsymbol{c}_{k, i}=\frac{\alpha_{k} \boldsymbol{h}_{\mathrm{LOS}, k}+\beta_{k} \boldsymbol{U}_{k} \boldsymbol{\Lambda}_{k}^{\frac{1}{2}} \boldsymbol{v}_{i}}{\left\|\alpha_{k} \boldsymbol{h}_{\mathrm{LOS}, k}+\beta_{k} \boldsymbol{U}_{k} \boldsymbol{\Lambda}_{k}^{\frac{1}{2}} \boldsymbol{v}_{i}\right\|}\right\},
$$

where $i=1, \ldots, 2^{B}$, and $\boldsymbol{v}_{i}$ 's $\in \mathbb{C}^{r_{k} \times 1}$ are the RVQ codewords for the conventional isotropic RVQ codebook $\mathcal{V}_{\text {i. i. d. }}$

\section{Quantized Two-Stage Precoder}

Motivated by the fact that high dimensional analog beamformer could get away with statistical CSIT, a two-stage precoder with a reduced dimensionality codebook design is developed herein. Namely, the analog beamformer stage is designed based on the knowledge of statistical CSIT to steer the beams towards the statistical dominant eigendirections of the desired users while eliminating the impact of multiuser interference in the statistical sense, whereas the baseband precoder stage eliminates the multiuser interference by utilizing only a finite-rate quantized CSI of the reduced dimensionality effective channel. More specifically, the high dimensionality analog beamformer is designed by leveraging the channel mean and covariance statistics, namely $\boldsymbol{h}_{\mathrm{LOS}, k}, \boldsymbol{\Sigma}_{k}, K_{k}, \rho \forall k \in\{1,2, \ldots, K\}$, while the digital precoder design utilizes a quantized CSI of the reduced dimensionality CSIT of the effective channel $\widehat{\boldsymbol{H}}_{\mathrm{eff}}=\widehat{\boldsymbol{V}^{\dagger} \boldsymbol{H}}$. Hence, in contrast to (18) and (20), where the BS estimates and quantizes $N_{B S} \times 1$ instantaneous CSIT elements for each user's channel $\widehat{\boldsymbol{h}}_{k}$, in this section, each user terminal estimates and quantizes only $N_{R F} \times 1$ instantaneous CSIT elements for the effective channel $\widehat{\boldsymbol{h}}_{\mathrm{eff}, k}=\widehat{\boldsymbol{V}^{\dagger} \boldsymbol{h}_{k}}$ instead, where $N_{R F} \ll N_{B S}$. Then, each user feeds back the nearest codeword index to the BS via a finite-rate feedback channel. This solution offers a huge overhead reduction for the estimation and feedback of the instantaneous CSIT as the downlink training and uplink feedback overhead scales down to the number of RF chains (i.e., users) rather than the massive number of BS antennas.

Therefore, based on the quantized fed back CSIT in the two-stage hybrid precoding, the $k^{\text {th }}$ user's optimal two-stage baseband precoder is given as

$$
\boldsymbol{w}_{k}^{\star}=\frac{\left(\boldsymbol{V}^{\dagger} \boldsymbol{V}+\rho \sum_{j \neq k} \widehat{\boldsymbol{h}}_{\mathrm{eff}, j} \widehat{\boldsymbol{h}}_{\mathrm{eff}, j}^{\dagger}\right)^{-1} \widehat{\boldsymbol{h}}_{\mathrm{eff}, k}}{\left\|\boldsymbol{V}\left(\boldsymbol{V}^{\dagger} \boldsymbol{V}+\rho \sum_{j \neq k} \widehat{\boldsymbol{h}}_{\mathrm{eff}, j} \widehat{\boldsymbol{h}}_{\mathrm{eff}, j}^{\dagger}\right)^{-1} \widehat{\boldsymbol{h}}_{\mathrm{eff}, k}\right\|} .
$$

The optimal $k^{\text {th }}$ column for the analog beamformer is written as

$$
\boldsymbol{v}_{k}^{\star}=\boldsymbol{u}_{\max }\left[\left(\boldsymbol{I}_{N_{B S}}+\rho \sum_{j \neq k} \boldsymbol{R}_{j}\right)^{-1} \boldsymbol{R}_{k}\right] .
$$

The effective CQI $\left\|\boldsymbol{h}_{\mathrm{eff}, k}\right\|$, where $\boldsymbol{h}_{\mathrm{eff}, k}=\boldsymbol{V}^{\dagger} \boldsymbol{h}_{k}$, is assumed perfectly known at the BS, while the effective CDI $\widetilde{\boldsymbol{h}}_{\mathrm{eff}, k}=\frac{\boldsymbol{h}_{\mathrm{eff}, k}}{\left\|\boldsymbol{h}_{\mathrm{eff}, k}\right\|}$ is quantized to the nearest codeword $\boldsymbol{c}_{\text {eff, } k, \pi(k)}$ in the codebook $\mathcal{C}_{\text {eff, } k}$, where the codeword index for the $k^{\text {th }}$ user $\pi(k)$ is given as

$$
\pi(k)=\underset{\boldsymbol{c}_{\mathrm{eff}, k, i} \in \mathcal{C}_{\mathrm{eff}, k}}{\arg \max }\left|\widetilde{\boldsymbol{h}}_{\mathrm{eff}, k}^{\dagger} \boldsymbol{c}_{\mathrm{eff}, k, i}\right|^{2} .
$$

The quantization index for the $k^{\text {th }}$ user $\pi(k)$ is then fed back to the BS via a finite-rate of $B$ feedback bits. The BS utilizes this index to obtain the quantized CSIT which is given as $\widehat{\boldsymbol{h}}_{\mathrm{eff}, k}=\left\|\boldsymbol{h}_{\mathrm{eff}, k}\right\| \boldsymbol{c}_{\mathrm{eff}, k, \pi(k)}$. The $k^{\text {th }}$ column for the analog beamformer $\boldsymbol{V}$ is designed based on statistical CSIT according to (22), whereas the $k^{\text {th }}$ column of the baseband precoder $\boldsymbol{W}$ is designed based on the quantized CSIT $\widehat{\boldsymbol{H}}_{\mathrm{eff}}=\left[\widehat{\boldsymbol{h}}_{\mathrm{eff}, 1}, \widehat{\boldsymbol{h}}_{\mathrm{eff}, 2}, \ldots, \widehat{\boldsymbol{h}}_{\mathrm{eff}, K}\right]$ at the BS according to $(21)$. 


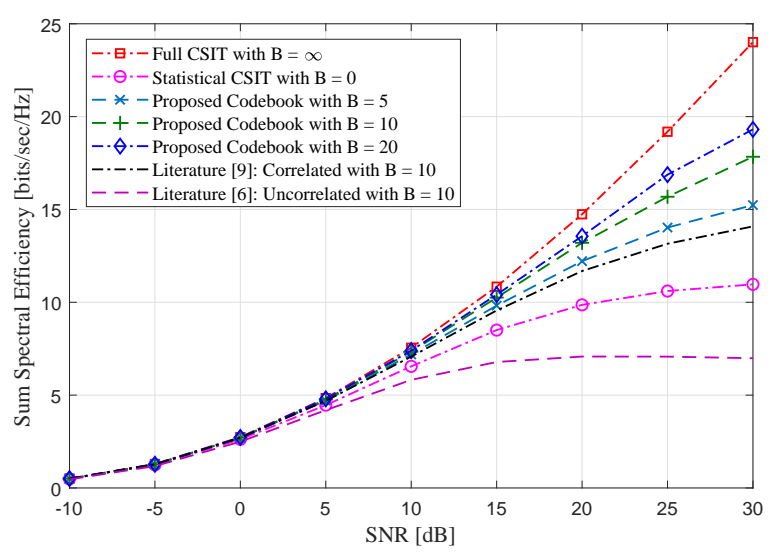

Figure 2: The sum spectral efficiency versus the SNR for $K=N_{B S}=4$, i.e., fully digital scenario.

Motivated by the proposed adaptive statistical codebook in (20), and the two-stage baseband precoder and analog beamformer in (21), (22), we introduce a reduced dimensionality adaptive statistical codebook as follows

$$
\mathcal{C}_{\mathrm{eff}, k}=\left\{\boldsymbol{c}_{\mathrm{eff}, k, i}=\frac{\boldsymbol{V}^{\dagger}\left(\alpha_{k} \boldsymbol{h}_{\mathrm{LOS}, k}+\beta_{k} \boldsymbol{U}_{k} \boldsymbol{\Lambda}_{k}^{\frac{1}{2}} \boldsymbol{v}_{i}\right)}{\left\|\boldsymbol{V}^{\dagger}\left(\alpha_{k} \boldsymbol{h}_{\mathrm{LOS}, k}+\beta_{k} \boldsymbol{U}_{k} \boldsymbol{\Lambda}_{k}^{\frac{1}{2}} \boldsymbol{v}_{i}\right)\right\|}\right\},
$$

where $i=1, \ldots, 2^{B}$. Note that in contrast to $(20)$ where the required number of feedback bits scales with $r_{k}$, the number of feedback bits $B$ here scales with $\min \left(r_{k}, N_{R F}\right)$ instead, according to $B=O\left(\frac{\min \left(r_{k}, N_{R F}\right)-1}{3} \rho_{\mathrm{dB}}\right)$.

\section{NUMERICAL RESULTS}

In this section, numerical results are provided to evaluate the performance of the analog beamformers and baseband precoders under finite-rate quantized CSIT with the transformed (shifted and skewed) RVQ codebook framework. The channel statistics information (mean and covariance) are assumed perfectly known at the users and BS, while the instantaneous CSI is quantized and fed back to the BS via low-rate limited feedback channel. The traditional isotropic (uncorrelated) RVQ codebook in [6] and skewed (correlated) RVQ codebook in [9] are considered for benchmarking. According to the one-ring local scattering correlation model, the $(l, m)^{\text {th }}$ entry for the $k^{\text {th }}$ user covariance matrix $\boldsymbol{\Sigma}_{k}$, is given as [14]

$$
\left[\boldsymbol{\Sigma}_{k}\right]_{l, m}=\frac{1}{2 \Delta_{k}} \int_{\phi_{k}-\Delta_{k}}^{\phi_{k}+\Delta_{k}} e^{-j \frac{2 \pi d}{\lambda}(m-l) \sin \left(\theta_{k}\right)} d \theta_{k}
$$

where $\Delta_{k}$ denotes the $k^{\text {th }}$ user azimuth angular spread, $\phi_{k}$ is the $k^{\text {th }}$ user central azimuth angle, and $\theta_{k}$ is the $k^{\text {th }}$ user actual AoD, distributed according to $\theta_{k} \sim$ $\mathcal{U}\left(\phi_{k}-\Delta_{k}, \phi_{k}+\Delta_{k}\right)$. In our simulations, we set $\phi_{k} \sim$ $\mathcal{U}\left(-\frac{\pi}{2}, \frac{\pi}{2}\right), \Delta_{k}=20^{\circ} \forall k, d=\frac{\lambda}{2}$, and $K_{k}=10 \mathrm{~dB} \forall k$.

In Fig. 2, the achievable sum spectral efficiency in (6) of the analog beamformer in (18) and digital precoder in (17) are shown against the SNR for the proposed adaptive

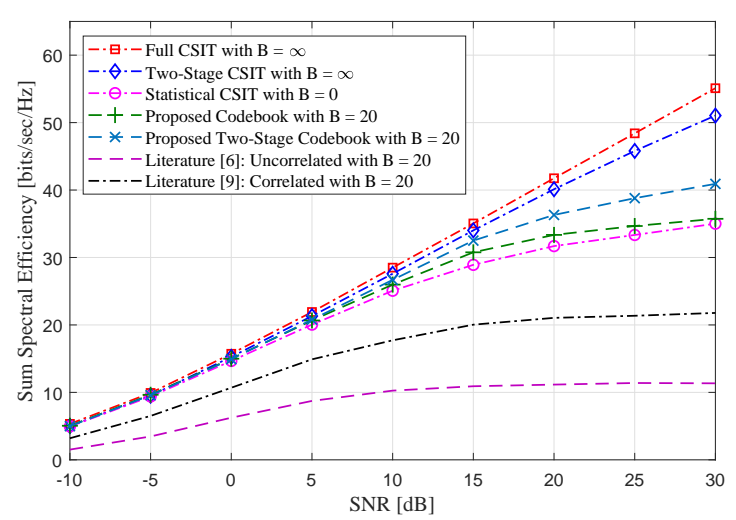

Figure 3: The sum spectral efficiency versus the SNR for $N_{B S}=64$ and $K=4$.

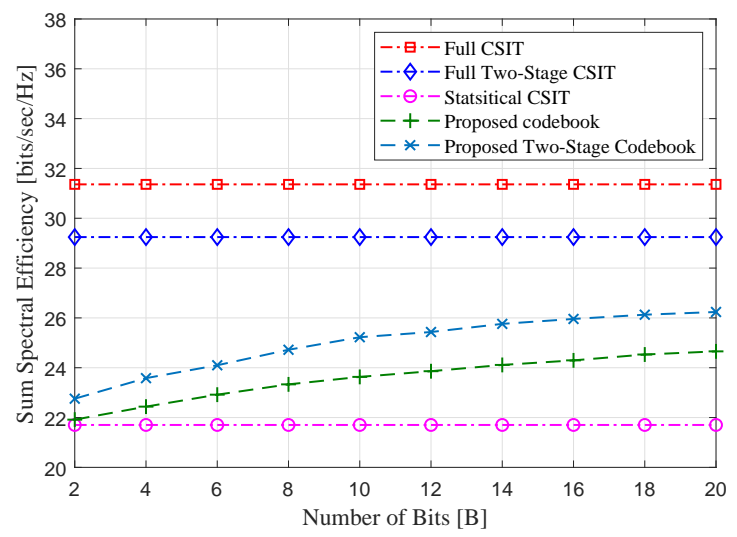

Figure 4: The sum spectral efficiency versus the number of feedback bits $B$ for $N_{B S}=16, K=4$, and $\rho=20 \mathrm{~dB}$.

statistical codebook in (20), for $N_{B S}=4$ antennas, $K=4$ users, and for different numbers of feedback bits $B$. For comparison purposes, the sum spectral efficiency for the case of perfect $(B=\infty)$ and statistical $(B=0)$ CSIT are included based on the analog beamformers in (10), (14), and digital precoders in (9), (12), respectively. It is clear that the proposed adaptive statistical codebook outperforms the conventional codebooks since the proposed codebook finely captures the channel subspace and does not blindly and uniformly quantize the whole space. The selection of random (channel independent) codebooks results in substantial quantization error at low to moderate number of feedback bits, while an infinitely large number of feedback bits is impractical.

Fig. 3 shows the impact of quantized massive MIMO channel on the sum spectral efficiency performance. Since the number of feedback bits needs to scale linearly with the number of BS antennas and SNR (in decibels) to control the quantization distortion, it is evidently observed that even with $B=20$ bits, no substantial performance improvement is seen by utilizing the proposed codebook in (20) beyond that of the statistical CSIT. This is due to the fact that $B$ needs to scale as $O\left(\frac{r_{k}-1}{3} \rho_{\mathrm{dB}}\right)$. However, with the twostage analog beamformer and baseband precoder in (22) 
and (21), respectively, and the introduced reduced dimensionality adaptive statistical codebook in (24), a significant sum spectral efficiency improvement is noticed beyond that of the statistical CSIT and the adaptive statistical codebook in (20). This is due to the fact that each user terminal quantizes a reduced dimensionality effective channel, thus substantially reducing the required number of bits $B$ to scale as $O\left(\frac{\min \left(r_{k}, N_{R F}\right)-1}{3} \rho_{\mathrm{dB}}\right)$ instead. Hence, at the $k^{\text {th }}$ user, rather than quantizing $\widetilde{\boldsymbol{h}}_{k} \in \mathbb{C}^{N_{B S} \times 1}$ which consumes huge feedback overhead, the $k^{\text {th }}$ user quantizes only $\widetilde{\boldsymbol{h}}_{\text {eff, } k} \in \mathbb{C}^{N_{R F} \times 1}$ instead.

In Fig. 4, the achievable sum spectral efficiency is shown against the number of feedback bits $B$, with $N_{B S}=16$ antennas, $K=4$ users, and $\rho=20 \mathrm{~dB}$. It is observed that the sum spectral efficiency for the proposed codebooks in (20) and (24) improves with the number of feedback bits $B$, while also seen that the reduced dimensionality adaptive statistical codebook in (24) outperforms its adaptive statistical codebook counterpart in (20). For instance, in order to attain a sum spectral efficiency of $\mathrm{R}_{\text {sum }}=24.1$ $\mathrm{bits} / \mathrm{sec} / \mathrm{Hz}$, the number of feedback bits needed is $B=14$ bits for the adaptive statistical codebook in (20), whereas it is only $B=6$ bits for the reduced dimensionality adaptive statistical codebook in (24). This is a substantial reduction not only in the feedback overhead and codebook size (which is reduced from $2^{14}$ to $2^{6}$ ), but also in the required training sequences, and search complexity (computational burden).

\section{CONCLUSIONS}

A new adaptive statistical codebook design for FDD multiuser MIMO systems was proposed for correlated Rician fading channels with arbitrary rank by leveraging the longterm channel statistics. The proposed codebook achieves a significant feedback overhead reduction over traditional codebooks. Furthermore, since the number of feedback bits scales linearly with the number of BS antennas, this codebook results in overwhelming feedback overhead in FDD massive MIMO systems. Therefore, motivated by the twostage precoding scheme where the analog beamformer was designed based on statistical CSIT, whereas the baseband precoder was determined by low-rate quantized CSI of the reduced dimensionality effective channel, a reduced dimensionality adaptive statistical codebook was developed. Our solutions efficiently reduce the acquisition overhead to scale with the number of RF chains rather than the number of BS antennas.

\section{APPENDIX A}

PROOF OF EQUATION (10)

Once substituting (9) into (7), the optimal $k^{\text {th }}$ column of the analog beamformer $\boldsymbol{V}^{\star}$ is simplified as

$$
\begin{aligned}
& \boldsymbol{v}_{k}^{\star}=\underset{\boldsymbol{v}_{k}}{\arg \max } \boldsymbol{h}_{k}^{\dagger} \boldsymbol{V}\left(\boldsymbol{V}^{\dagger} \boldsymbol{V}+\rho \sum_{j \neq k} \boldsymbol{V}^{\dagger} \boldsymbol{h}_{j} \boldsymbol{h}_{j}^{\dagger} \boldsymbol{V}\right)^{-1} \boldsymbol{V}^{\dagger} \boldsymbol{h}_{k} \\
= & \underset{\boldsymbol{v}_{k}}{\arg \max } \operatorname{Tr}\left(\left(\boldsymbol{V}^{\dagger} \boldsymbol{V}+\rho \sum_{j \neq k} \boldsymbol{V}^{\dagger} \boldsymbol{h}_{j} \boldsymbol{h}_{j}^{\dagger} \boldsymbol{V}\right)^{-1} \boldsymbol{V}^{\dagger} \boldsymbol{h}_{k} \boldsymbol{h}_{k}^{\dagger} \boldsymbol{V}\right) .
\end{aligned}
$$

This is a quotient trace problem and is solved by the generalized eigenvalue decomposition method [15]

$$
\boldsymbol{h}_{k} \boldsymbol{h}_{k}^{\dagger} \boldsymbol{v}_{k i}=\lambda_{k, i}\left(\boldsymbol{I}_{N_{B S}}+\rho \sum_{j \neq k} \boldsymbol{h}_{j} \boldsymbol{h}_{j}^{\dagger}\right) \boldsymbol{v}_{k i},
$$

where $\boldsymbol{v}_{k i}$ is the $i^{\text {th }}$ leading eigenvector corresponding to $\lambda_{k, i}$, the $i^{\text {th }}$ strongest eigenvalue of the generalized eigenvalue decomposition for the $k^{\text {th }}$ user.

Therefore, since $\left(\boldsymbol{I}_{N_{B S}}+\rho \sum_{j \neq k} \boldsymbol{h}_{j} \boldsymbol{h}_{j}^{\dagger}\right) \quad$ is invertible, $\boldsymbol{v}_{k}^{\star}$ is the leading eigenvector corresponding to the strongest generalized eigenvalue of the matrix $\quad\left(\boldsymbol{I}_{N_{B S}}+\rho \sum_{j \neq k} \boldsymbol{h}_{j} \boldsymbol{h}_{j}^{\dagger}\right)^{-1} \boldsymbol{h}_{k} \boldsymbol{h}_{k}^{\dagger}, \quad$ i.e., $\boldsymbol{v}_{k}^{\star}=\boldsymbol{u}_{\max }\left[\left(\boldsymbol{I}_{N_{B S}}+\rho \sum_{j \neq k} \boldsymbol{h}_{j} \boldsymbol{h}_{j}^{\dagger}\right)^{-1} \boldsymbol{h}_{k} \boldsymbol{h}_{k}^{\dagger}\right]$, which results in (10). This concludes the proof.

\section{REFERENCES}

[1] A. Alkhateeb, J. Mo, N. Gonzalez-Prelcic, and R. W. Heath, Jr., "MIMO precoding and combining solutions for millimeter-wave systems," IEEE Commun. Mag., vol. 52, no. 12, pp. 122-131, Dec. 2014.

[2] A. Alkhateeb, G. Leus, and R. W. Heath, Jr., "Limited feedback hybrid precoding for multi-user millimeter wave systems," IEEE Trans. Wireless Commun., vol. 14, no. 11, pp. 6481-6494, Nov. 2015.

[3] M. Dai and B. Clerckx, "Multiuser millimeter wave beamforming strategies with quantized and statistical CSIT," IEEE Trans. Wireless Commun., vol. 16, no. 11, pp. 7025-7038, Nov. 2017.

[4] S. Park, J. Park, A. Yazdan, and R. W. Heath, Jr., "Exploiting spatial channel covariance for hybrid precoding in massive MIMO systems," IEEE Trans. Signal Process., vol. 65, no. 14, pp. 3818-3832, Jul. 2017.

[5] A. F. Molisch, V. V. Ratnam, S. Han, Z. Li, S. L. H. Nguyen, L. Li, and K. Haneda, "Hybrid beamforming for massive MIMO: A survey," IEEE Commun. Mag., vol. 55, no. 9, pp. 134-141, Sep. 2017.

[6] N. Jindal, "MIMO broadcast channels with finite-rate feedback," IEEE Trans. Inf. Theory, vol. 52, no. 11, pp. 5045-5060, Nov. 2006.

[7] A. Adhikary, J. Nam, J. Ahn, and G. Caire, "Joint spatial division and multiplexing - The large-scale array regime," IEEE Trans. Inf. Theory, vol. 59, no. 10, pp. 6441-6463, Oct. 2013.

[8] B. Lee, J. Choi, J. Seol, D. J. Love, and B. Shim, "Antenna grouping based feedback compression for FDD-based massive MIMO systems," IEEE Trans. Commun., vol. 63, no. 9, pp. 3261-3274, Sep. 2015.

[9] D. J. Love and R. W. Heath, Jr., "Limited feedback diversity techniques for correlated channels," IEEE Trans. Veh. Technol., vol. 55, no. 2, pp. 718-722, Mar. 2006.

[10] W. Shen, L. Dai, B. Shim, Z. Wang, and R. W. Heath, Jr., "Channel feedback based on AoD-adaptive subspace codebook in FDD massive MIMO systems," IEEE Trans. Commun., vol. 66, no. 11, pp. 52355248, Nov. 2018.

[11] F. Sohrabi and W. Yu, "Hybrid digital and analog beamforming design for large-scale antenna arrays," IEEE J. Sel. Topics Signal Process., vol. 10, no. 3, pp. 501-513, Apr. 2016.

[12] D. Kim, G. Lee, and Y. Sung, "Two-stage beamformer design for massive MIMO downlink by trace quotient formulation," IEEE Trans. Commun., vol. 63, no. 6, pp. 2200-2211, Jun. 2015.

[13] M. Sadek, A. Tarighat, and A. H. Sayed, "A leakage-based precoding scheme for downlink multi-user MIMO channels," IEEE Trans. Wireless Commun., vol. 6, no. 5, pp. 1711-1721, May 2007.

[14] A. Adhikary, E. Al Safadi, M. K. Samimi, R. Wang, G. Caire, T. S. Rappaport, and A. F. Molisch, "Joint spatial division and multiplexing for mm-wave channels," IEEE J. Sel. Areas Commun., vol. 32, no. 6, pp. 1239-1255, June 2014

[15] H. Wang, S. Yan, D. Xu, X. Tang, and T. Huang, "Trace ratio vs. ratio trace for dimensionality reduction," in Proc. IEEE CVPR, June 2007, pp. 1-8. 